\title{
Generation of amplified spontaneous emission from rare-earth complexes dispersed in phenol + AOT self-assembled
}

\section{organogels}

Katsura Nishiyama, ${ }^{\mathrm{a}, *}$ Yasuhiro Watanabe, ${ }^{\mathrm{a}}$ Takashi Harada, ${ }^{\mathrm{a}}$ Kenji Kamada, ${ }^{\mathrm{b}}$ and Hideki Kawai $^{\mathrm{c}, ~ *}$

${ }^{\text {a }}$ Faculty of Education, Shimane University, Matsue 690-8504, Japan

${ }^{\mathrm{b}}$ Research Institute for Ubiquitous Energy Devices, National Institute of Advanced Industrial Science and Technology (AIST), Ikeda, Osaka 563-8577, Japan

${ }^{\mathrm{c}}$ Department of Applied Chemistry and Biochemical Engineering, Graduate School of Engineering, Shizuoka University, Hamamatsu 432-8011, Japan

*Corresponding authors:

Katsura Nishiyama

E-mail: katsura_nishiyama@edu.shimane-u.ac.jp

TEL/FAX: +81-852-32-9832

Hideki Kawai

E-mail: rohkawa@ipc.shizuoka.ac.jp

TEL/FAX: +81-53-478-1653

\section{Abstract}

The rare-earth complex $\mathrm{Eu}(\mathrm{hfa})_{3}$ (phen) is dispersed in the self-assembled organogel of p-chlorophenol and surfactant AOT. The time profiles of the emission intensity observed from the complex-organogel system suggest the possible generation of amplified stimulated emission when excited with a strong excitation pulse. As the host material, the organogel can support eight times as much of the complex along with better emission quantum yield. A theoretical estimation of the laser 
gain factor of the complex-organogel also suggested that this system might have application in laser systems.

\section{Keywords}

organogel, soft material, self-healing material, Eu complex, laser material 


\section{Introduction}

Self-assembled organogels composed of small organic molecules have been used to hosting materials that contain rare-earth complexes [1,2], nanoparticles [3,4], nanofibers [5], nanorings [6], or lipophilic compounds [7]. Such doping processes rely on the organogel network structure, where interactions between the network and dopants are critical. The network structure fluctuates either in the temporal and spatial phase, which is characteristic of soft materials. An earlier paper of ours discussed the distribution of the time constant of the structural fluctuation of organogels in the nanosecond region [8].

An advantage of using organogels as host materials is that they easily reformat when assembled in a solid vessel. The self-healing of organogels is also attractive [6,9]. However, organogels may exhibit turbidity owing to their microscopic structure, which could hinder applications to optical devices.

In a previous study, we dispersed $\mathrm{Eu}^{3+}$ and $\mathrm{Tb}^{3+}$ complexes in an optically transparent phenol + AOT organogel [2]. The organogel structure, which was suggested by Simmons and coworkers [10], is shown in Chart 1a. These complex-organogel systems have been applied to additive emission color-tuning in the visible region [2]. Another advantage of the phenol + AOT organogel as host material is that we can disperse in it the $\mathrm{Eu}(\mathrm{hfa})_{3}(\mathrm{phen})$ complex, which is 8 times denser than the liquid, along with higher emission quantum yield $\Phi_{\mathrm{f}}$. Table 1 lists $\Phi_{\mathrm{f}}$ and the maximum concentration of $\mathrm{Eu}(\mathrm{hfa})_{3}$ (phen) $c_{\max }$. Note that hexafluoroacetylacetone (hfa) and 1,10-phenanthroline (phen) were chosen as the ligands for this complex, as shown in Chart 1b. We have also reported that the complexes emit independently even in the organogel environment, as the organogel network can separate the mutual interactions between the complexes. Furthermore, it has been shown that the interplay between the organogel architecture and the complexes hardly affects the excited state of the rare earths [2].

In this study, we have used the properties of the organogel in a laser material by dispersing $\mathrm{Eu}(\mathrm{hfa})_{3}(\text { phen) in the organogel. Previously, we dispersed Eu(hfa })_{3}(\mathrm{TPPO})_{2}[11]$ and its derivatives 
possessing various ligands [12] into thin polystyrene films, where the ligand TPPO represents triphenylphospine oxide, and monitored the time profiles of the emission intensity $I(t)$. The $I(t)$ profiles clearly depend on the laser excitation-pulse energy $E_{\mathrm{ex}}$, the results of which have been ascribed to the generation of amplified spontaneous emission (ASE), which is a kind of cavity-less laser action and the light amplification process of spontaneous emission (i.e. fluorescence) by stimulated emission through the inverted population generated by the photoexcitation [13]. According to the previous reports $[11,12], \mathrm{Eu}^{3+}$ complexes have thus been shown to be candidate laser materials when dispersed in thin films. In the present study, we will show that the organogel can also be used as an environment for laser materials, while maintaining the previously described attractive characteristics of soft materials. The estimated laser gain factor of the organogel system suggests potential applicability to laser materials. We hereafter denote a system where a Eu complex is dispersed in organogel, film, and liquid solvent as “Eu@gel," “Eu@film,” and “Eu@liq," respectively.

\section{Materials and methods}

\subsection{Materials.}

The synthesis of the phenol + AOT organogel and Eu(hfa $)_{3}($ phen) were previously reported $[2,8]$. We followed Simmons et al. for the organogel synthesis [10]. Briefly, $0.14 \mathrm{~mol} \mathrm{dm}^{-3}$ $p$-chlorophenol and $0.10 \mathrm{~mol} \mathrm{dm}^{-3}$ AOT (bis(2-ethylhexyl)sulfosuccinate) dissolved in $m$-xylene solvent were combined to form the gel at room temperature (298 K). To prepare Eu@gel, $\mathrm{Eu}(\mathrm{hfa})_{3}$ (phen) was added to the organogel and then both were heated up to $353 \mathrm{~K}$ in a water bath to obtain an isotropic solution. Subsequently, they were cooled to obtain a gel phase, and the heating and cooling cycles were repeated several times. A Eu(hfa) $)_{3}($ phen) solution in $m$-xylene was used as

Eu@liq. Regarding the dispersing concentration of Eu(hfa) $)_{3}(\mathrm{phen}), c_{\max }$ was chosen either for Eu@gel and Eu@gel, as given in Table 1. 


\subsection{Measurements of the photochemical and optical properties.}

The steady-state absorption and emission, or excitation, spectra were recorded with a Hitachi U2800 spectrometer and a Jasco FP-6500 spectrofluorometer, respectively. A Jasco ILF-533 integrating sphere was employed for the $\Phi_{\mathrm{f}}$ measurements. A DR-A1 (Atago) Abbe refractometer was used to measure the refractive index. The time-dependent measurements were performed with the equipment we had previously used [11,12]. The third harmonics of a Nd:YAG laser from B. M. Industries $[10 \mathrm{~Hz}$, full-width-at-half-maximum $(\mathrm{fwhm}) \approx 9 \mathrm{~ns}]$ was used as the excitation light source, with wavelength $\lambda_{\mathrm{ex}}=355 \mathrm{~nm}$. As a result, the $\lambda_{\mathrm{ex}}=355 \mathrm{~nm}$ for the $I(t)$ measurements gives rise to the photoexcitation of the hfa ligand, followed by energy transfer to $\mathrm{Eu}^{3+}$ that causes emission [2]. Using a cylindrical lens, the laser beam was focused onto the front side of a foursquare quartz cuvette at normal incidence. The beam travelled $2 \mathrm{~mm}$ inside the sample. The emission was monitored from the side direction with a photomultiplier (Hamamatsu R928), which formed a perpendicular configuration between the excitation pulse and the emission to be monitored. A monochromator was used to collect the ${ }^{5} \mathrm{D}_{0}-{ }^{7} \mathrm{~F}_{2}$ emission of $\mathrm{Eu}^{3+}$ at the maximum of around $614 \mathrm{~nm}$. The $I(t)$ profiles were recorded with a digital oscilloscope and a sampling duration of $1 \mathrm{GHz}$.

\section{Results and discussion}

\subsection{The steady-state emission spectra of $\boldsymbol{E u} @$ liq and $\boldsymbol{E u} @ g e l$.}

Figure 1 compares the emission spectra of Eu@liq using $m$-xylene and $\mathbf{E u} @$ gel for $\lambda_{\mathrm{ex}}=355$ nm. The emission spectra of Eu@liq and Eu@ gel were independent of $\lambda_{\text {ex }}$. The spectral maximum of Eu@gel appears at $614 \mathrm{~nm}$, which is $2 \mathrm{~nm}\left(53 \mathrm{~cm}^{-1}\right)$ red-shifted compared with that of Eu@liq (612 $\mathrm{nm})$. We have also found that the fwhm of the Eu@gel spectrum corresponds to $127 \mathrm{~cm}^{-1}$, which is about $50 \%$ broader than that of Eu@liq $\left(80 \mathrm{~cm}^{-1}\right)$. For precision, the ${ }^{7} \mathrm{~F}_{2}$ level of $\mathrm{Eu}^{3+}$ was five-fold degenerate the ligands resolved the degeneracy $[12,14]$. We can therefore ascribe the spectral red 
shift observed in Eu@gel to environmental differences around Eu(hfa $)_{3}(\mathrm{phen})$, compared with the organogel and liquid. The organogel environment may produce more inhomogeneity, which causes broader fwhm in the emission spectrum.

\subsection{The emission time profiles and the excitation pulse energy.}

In this subsection, we discuss the $I(t)$ profiles detected with varying $E_{\mathrm{ex}}$, similarly to previous studies [11,12]. Figure 2 displays the $I(t)$ profiles for Eu@liq and Eu@gel, which can be fitted with a bi-exponential function

$$
I(t)=A_{1} \exp \left(-t / \tau_{1}\right)+A_{2} \exp \left(-t / \tau_{2}\right)
$$

where $\tau_{i}(i=1$ or 2$)$ stands for the lifetime and $A_{i}$ denotes the pre-exponential factor with $A_{1}+A_{2}=1$. Table 2 summarizes the quantities determined with Eq. (1) by using the least square method. The $I(t)$ profile of Eu@liq at $E_{\text {ex }}=0.02 \mathrm{~mJ}$ was not detected because of insufficient pulse energy.

Figure 2a shows that the $I(t)$ profiles for Eu@liq at $0.05-0.2 \mathrm{~mJ}$ mostly overlap with each other and the lifetime is around $\tau_{2}=1.5 \mathrm{~ms}$. This $\tau_{2}$ value is assigned to the lifetime of Eu(hfa $)_{3}(\mathrm{phen})$ in $m$-xylene solvent. When we increase $E_{\text {ex }}$ to $0.5 \mathrm{~mJ}$ or higher, the $I(t)$ profiles were fitted well with a biexponential function. In Table 2, the $A_{2}$ value for Eu @liq drops drastically between $E_{\mathrm{ex}}=0.2-0.5$ mJ. This phenomenon is due to the appearance of the fast component, with its amplitude of $A_{1}$. We have assigned the slower component $\tau_{2}$ also to the intrinsic lifetime of $\mathrm{Eu}(\mathrm{hfa})_{3}$ (phen) for each $E_{\mathrm{ex}}$ because $\tau_{2}$ varies only by $\sim 10 \%$ compared to the lifetime for $E_{\mathrm{ex}}$ of $2.0 \mathrm{~mJ}$ or less. Two possibilities can explain the appearance of the fast component $\tau_{1}$ : ASE and the annihilation process, where one excited molecule interact with another excited molecule because of high concentration of the excited species. Further study is needed to clarify the origin of $\tau_{1}$ of Eu @liq; however, that appears at much higher $E_{\mathrm{ex}}$ compared to $\mathbf{E u} @$ gel regardless of the origin. 
On the other hand, the $I(t)$ profiles for Eu@gel in Figure $2 \mathrm{~b}$ are much more complicated. A fast component was observed even with an excitation energy as low as $E_{\mathrm{ex}}=0.02 \mathrm{~mJ}$. Looking closely, both $\tau_{1}$ and $\tau_{2}$ become dramatically faster when we increase $E_{\mathrm{ex}}$, whereas $A_{1}$ becomes dominant.

As an indication of the total photon number emitting from the sample upon excitation, we introduce the parameter $J\left(E_{\mathrm{ex}}\right)$

$$
J\left(E_{\mathrm{ex}}\right)=\int_{t=0}^{\infty} I(t) d t
$$

To estimate $J\left(E_{\text {ex }}\right)$ using Eq. (2), the absolute intensity of the $I(t)$ profiles was scaled in accordance with the sensitivity of the photomultiplier used in the measurements. Figure 3 shows the estimated $J\left(E_{\mathrm{ex}}\right)$, which is normalized as $J\left(E_{\mathrm{ex}}=2.0 \mathrm{~mJ}\right)=1.0$ for $\mathbf{E u} @$ gel. We have found that $J\left(E_{\mathrm{ex}}\right)$ for Eu@gel drastically increases when $E_{\text {ex }}$ becomes large. On the contrary, $J\left(E_{\text {ex }}\right)$ for $\mathbf{E u} @$ liq shows only a weak increase against $E_{\text {ex }}$. The trends of $J\left(E_{\text {ex }}\right)$ indicate that strong $E_{\text {ex }}$ increases the emitting photons for Eu@gel, whereas such an obvious increment is not applicable to Eu@liq.

We next focus our attention on how $E_{\mathrm{ex}}$ influences the pre-exponential factor $A_{1}$ in Eq. (1). Figure 4 plots $A_{1}$ for $\mathbf{E u} @ \mathbf{g e l}$ as a function of $E_{\mathrm{ex}}$. Also shown in Figure 4 is the corresponding data set for Eu@ film, which is taken from ref [11]. When $E_{\text {ex }}$ is increased, both plots increase as a general trend. It is commented that the large error bars for $\mathbf{E u} @$ gel data in the small $E_{\text {ex }}$ region is as a consequence of small signal intensity when detected. In Figure 4, we find a systematic offset $\Delta A_{1}$ between the data sets of Eu@gel and Eu@film, with $\Delta A_{1}=0.2-0.3$. Apart from $\Delta A_{1}$, the upward tendency with increasing $E_{\mathrm{ex}}$ for both data sets is similar. With regard to the Eu@ film, it has been proven that when $E_{\text {ex }}$ is $0.02 \mathrm{~mJ}$, the $I(t)$ profile is expressed by single exponential decay that causes $A_{1}=0$. Even with employing various ligands for $\mathbf{E u} @$ film, a similar trend regarding the increase in $A_{1}$ according to $E_{\text {ex }}$ has been previously reported [12].

In earlier reports $[11,12]$, we confirmed that $\mathbf{E u} @$ film provides ASE when excited with $E_{\text {ex }}$ of adequate magnitude. In ref $[11,12]$, the assignment of ASE is due to the systematic rise of $A_{1}$ and the 
appearance of the fast component $\tau_{1}$, both of which are associated with the increase in $E_{\mathrm{ex}}$. In this context, the observed emission behavior of Eu@gel can also be attributed to ASE. The upward tendency for $A_{1}$ in Figure 4 for $\mathbf{E u} @$ gel is similar to that of Eu@film. Furthermore, the emitting photon number measured using $J\left(E_{\mathrm{ex}}\right)$ dramatically increases with $E_{\mathrm{ex}}$. Such features are characteristic to Eu@gel and not applicable to Eu@liq.

Finally, we compare the microscopic environments of the organogel and liquid that support $\mathrm{Eu}(\mathrm{hfa})_{3}(\mathrm{phen})$. The $c_{\max }$ in Table 1 is the bulk concentration of $\mathrm{Eu}(\mathrm{hfa})_{3}(\mathrm{phen})$ in the gel or liquid environment. When liquid solvent is used, an isotropic solution is obtained. In contrast, we have reported that the majority of Eu(hfa) $)_{3}$ (phen) is localized in the exterior of the "fiber-bundle" structure of the organogel, as illustrated in Chart 1a [2]. Under such conditions, we roughly estimate the effective concentration $c_{\text {eff }}(\mathbf{E u} @$ gel $)$ of $\mathrm{Eu}(\mathrm{hfa})_{3}($ phen $)$ in the organogel. It is assumed that the complex is located within a space of $0.1 \mathrm{~nm}$ from the fiber bundle and solvent interface, estimated by the dimension of the complex. We used atomic force microscopy [15] to obtain the diameter of the fiber bundle and the density of the fiber bundle sustained in the solvent in the gel matrix. As a result, we obtain $c_{\text {eff }}(\mathbf{E u} @$ gel $) \approx 1 \mathrm{~mol} \mathrm{dm}{ }^{-3}$ that is $10^{4}$ times higher than the $c_{\text {max }}$ of Eu@ liq. Such a dense dispersion environment for $\mathrm{Eu}(\mathrm{hfa})_{3}(\mathrm{phen})$ in the organogel should lead to stronger interactions between the complexes, when photoexcited. This is plausible explanation for the biexponential lifetime of $\mathbf{E u} @$ gel even for small $E_{\text {ex }}$ gives rise to the offset $\Delta A_{1}$ between $\mathbf{E u} @$ gel and $\mathbf{E u} @$ liq, as shown in Figure 4. We suggest that such an environment specific to the organogel is likely responsible for the generation of ASE.

\subsection{Estimation of the laser gain factor for $\boldsymbol{E} \boldsymbol{u} @ g e l$.}

We estimate the laser gain factor for Eu@gel applied to a laser material. The gain factor $\gamma$ at the lasing frequency $v$ is calculated with [13] 


$$
\gamma(v)=\left(N_{1}-N_{2}\right) \frac{c^{2}}{8 \pi n^{2} v^{2} t_{\text {spont }}} g(v)
$$

where $N_{i}(i=1$ or 2$)$ expresses the atom number in state $i$. In this notation, states 1 and 2 respectively correspond to the ground and excited states of the emitting atom. $c, n$, and $t_{\text {spont }}$ denote the speed of light, the refractive index of the medium, and the spontaneous lifetime of the emitting atom, respectively. The last undefined term in Eq. (3) is $g(v)$, which is the spectral shape function of the transitions between states 1 and 2. The parameters in Eq. (3) were estimated according to the following steps. $v=4.88 \square 10^{14}$ rom the emission maximum of Eu @ gel at 614 nm, and $N_{1}-N_{2}=4.82$

$10^{17} \mathrm{~cm}^{-3}$ based on the comparison of the $\mathrm{Eu}^{3+}$ number for $\mathbf{E u} @$ gel in the optical cuvette and the photon density of the laser excitation pulse irradiated in the cuvette. $t_{\text {spont }}=1.5 \square 10^{-3} \mathrm{~s}$, which corresponds to $\tau_{2}$ of Eu @liq associated with the average data for $E_{\mathrm{ex}}=0.05-0.2 \mathrm{~mJ}$. In that region of $E_{\text {ex }}$, the $I(t)$ profiles decay with a single component. Moreover, $g(v)$ is approximately $4.54 \square 10^{-13} \mathrm{~s}$, taken from the inverse of the emission spectral bandwidth with maximum at $614 \mathrm{~nm}$ [13]. Relying on the result for Eu$@$ gel, $n=1.4963$, we obtained $\gamma(v) \approx 0.001 \mathrm{~cm}^{-1}$. This $\gamma(v)$ value corresponds to $1 \%$ amplification when light travels $1 \mathrm{~cm}$ inside the medium. Note that a conventional helium-neon laser has a gain of $\gamma_{\mathrm{HeNe}}(v) \approx 0.005 \mathrm{~cm}^{-1}[13]$.

We have shown above that the emission from $\mathbf{E u} @$ gel can be attributed to ASE, based on the $I(t)$ properties of Eu@film [11,12]. Along with the estimated $\gamma(v)$ above, we suggest that $\mathbf{E u} @$ gel might be useful as a laser medium.

\section{Summary and future perspective}

For this study, we dispersed the $\mathrm{Eu}(\mathrm{hfa})_{3}(\mathrm{phen})$ complex in an organogel of $p$-chlorophenol and AOT that spontaneously gelled in the nonpolar $m$-xylene solvent. The sample, Eu@gel, was excited by the third harmonics of a Nd:YAG laser $(355 \mathrm{~nm})$ and the time profile of the emission intensity $I(t)$ 
was monitored. The $I(t)$ profiles of Eu@gel were fitted with a biexponential function. With increasing pulse excitation energy $\left(E_{\mathrm{ex}}\right)$, the pre-exponential factor of the fast component $\left(A_{1}\right)$ increases and the lifetime $\left(\tau_{1}\right)$ markedly decreases $(0.06 \mathrm{~ms})$ compared with the usual lifetime of Eu complexes (1.5 ms). In earlier papers we had dispersed Eu(hfa $)_{3}(\mathrm{TPPO})_{2}$ [11] and its derivatives [12] in a polystyrene thin film, which we call Eu@film. The $E_{\mathrm{ex}}$-dependent feature of $A_{1}$ in $\mathbf{E u} @ \mathbf{u e l}$ is rather similar with that reported for the Eu@film, which has been assigned to the generation of amplified spontaneous emission (ASE). The total photon number emitting from Eu@gel increases as $E_{\text {ex }}$ increases, which also implies the presence of ASE. In contrast, such a feature was not detected in the $\mathrm{Eu}(\mathrm{hfa})_{3}($ phen) liquid solution.

On the other hand, we have estimated that the laser-gain factor $\gamma$ for $\mathbf{E u} @$ gel resulted in $\gamma \approx 0.001$ $\mathrm{cm}^{-1}$. This corresponds to $1 \%$ amplification when light travels $1 \mathrm{~cm}$ in the medium. In connection with our prior results on Eu@film,we suggest that Eu@gel can be also used as a laser material. If rare-earth complexes are realized as laser materials, one can obtain lasing wavelengths that are not available from conventional LD lasers, without relying on the wavelength-conversion technique. Furthermore, organogel materials can be easily reformatted when used in optical equipment. This is an advantage of using organogel materials as dispersing media.

We can disperse Eu(hfa) $)_{3}\left(\right.$ phen) as much as $8.0 \square 10^{-4} \mathrm{~mol} \mathrm{dm}^{-3}$ in this type of organogel. This is 8 times more than the saturation level of Eu(hfa) $)_{3}($ phen) dissolved in $m$-xylene. Furthermore, in the present organogel environment, Eu(hfa $)_{3}(\mathrm{phen})$ is localized in the exterior of the fiber-bundle structure [2]. We estimated the effective concentration $c_{\text {eff }}(\mathbf{E u} @$ gel $)$ of Eu(hfa) $)_{3}($ phen $)$ localized in the organogel architecture, $c_{\text {eff }}(\mathbf{E u} @ \mathbf{g e l})=1 \mathrm{~mol} \mathrm{dm}^{-3}$, which is $10^{4}$ times as much as that in organic solvents. Such a characteristic dense dispersion environment of $\mathrm{Eu}(\mathrm{hfa})_{3}(\mathrm{phen})$ in the organogel offers specific interactions between the complexes followed by photoexcitation. The $I(t)$ profile depending on $E_{\mathrm{ex}}$ is an example caused by such interactions, as shown in this study. Another merit of the specific organogel environment is that the emission quantum yield of Eu@gel $\left(\Phi_{\mathrm{f}}=0.22\right)$ is enhanced, compared with that of Eu@liq $\left(\Phi_{\mathrm{f}}=0.16\right)$. On the other hand, the interaction between the 
rare-earth complex and organogel structure may also be critical in such characteristic photochemical behavior. Further studies to clarify the dispersion structure of the complexes and the interaction mechanisms are needed.

\section{Acknowledgment}

We thank Professor Toshiyuki Takamuku at Saga University for the refractive index measurements. KN acknowledges financial support from JSPS KAKENHI (Grant Number 25410211), JST A-STEP (AS242Z01279M), the Mazda Foundation, and the Electric Technology

Research Foundation of Chugoku. We thank the Cooperative Research Project of Research Institute of Electronics, Shizuoka University.

\section{References}

[1] S. Dehn, K.W.K. Tong, R.G.C. Clady, D.M. Owen, K. Gaus, T.W. Schmidt, F. Braet, P. Thordarson, New J. Chem. 35 (2011) 1466.

[2] K. Nishiyama, Y. Watanabe, K. Watanabe, T. Harada, Chem. Lett. 41 (2012) 1697.

[3] B. Simmons, S. Li, V.T. John, G.L. McPherson, C. Taylor, D.K. Schwartz, K. Maskos, Nano Lett. 2 (2002) 1037.

[4] V.C. Edelsztein, E.A. Jares-Erijman, K. Müllen, P.H.D. Chenna, C.C. Spagnuolo, J. Mat. Chem. 2012 (2012) 21857.

[5] S.H. Kang, B.M. Jung, W.J. Kim, J.Y. Chang, Chem. Mater. 20 (2008) 5532.

[6] X. Yu, X. Cao, L. Chen, H. Lan, B. Liu, T. Yi, Soft Matter 8 (2012) 3329.

[7] K. Iwanaga, T. Sumizawa, M. Miyazaki, M. Kakemi, Int. J. Pharm. 388 (2010) 123.

[8] K. Nishiyama, K. Takata, K. Watanabe, H. Shigematsu, Chem. Phys. Lett. 529 (2012) 39.

[9] P. Mukhopadhyay, N. Fujita, A. Takada, T. Kishida, M. Shirakawa, S. Shinkai, Angew. Chem. Int. Ed. Engl. 49 (2010) 6338.

[10] B.A. Simmons, C.E. Taylor, F.A. Landis, V.T. John, G.L. McPherson, D.K. Schwartz, R. Moore, J. Am. Chem. Soc. 123 (2001) 2414.

[11] Y. Hasegawa, Y. Wada, S. Yanagida, H. Kawai, N. Yasuda, T. Nagamura, Appl. Phys. Lett. 83 (2003) 3599.

[12] K. Nakamura, Y. Hasegawa, H. Kawai, N. Yasuda, N. Kanehisa, Y. Kai, T. Nagamura, S. Yanagida, Y. Wada, J. Phys. Chem. A 111 (2007) 3029.

[13] A. Yariv, "Quantum Electronics", 4th Ed., Saunders College, Orland, 1991.

[14] M.H.V. Werts, R.T.F. Jukes, J.W. Verhoeven, Phys. Chem. Chem. Phys. 4 (2002) 1542.

[15] K. Nishiyama, K. Watanabe, Y. Watanabe, manuscript in preparation. 
Table 1. $c_{\max }$ and $\Phi_{\mathrm{f}}$ of $\mathrm{Eu}(\mathrm{hfa})_{3}$ (phen) dispersed in the organogel and organic solvent.

\begin{tabular}{lll}
\hline sample & $\begin{array}{c}\boldsymbol{c}_{\text {max }} \\
10^{-4} \mathrm{~mol} \mathrm{dm}^{-3}\end{array}$ & $\Phi_{\mathrm{f}}$ \\
& $8.0^{\mathrm{a})}$ & 0.22 \\
\hline Eu@gel & $1.0^{\mathrm{a})}$ & 0.16 \\
\hline $\mathbf{E u} @$ liq $^{\text {b) }}$ & & \\
\hline
\end{tabular}

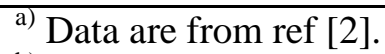

b) Dispersed in $m$-xylene solvent.

Table 2. Lifetimes and pre-exponential factors. ${ }^{\text {a) }}$

\begin{tabular}{llll}
\hline \hline sample & $E_{\text {ex }} / \mathrm{mJ}$ & $\tau_{1} / \mathrm{ms}\left(A_{1}\right)$ & $\tau_{2} / \mathrm{ms}\left(A_{2}\right)$ \\
\hline \hline Eu@liq & $0.05^{\mathrm{b}}$ & - & $1.54 \pm 0.01(1.00)$ \\
& $0.1^{\mathrm{b})}$ & - & $1.56 \pm 0.01(1.00)$ \\
& $0.2^{\mathrm{b})}$ & - & $1.48 \pm 0.01(1.00)$ \\
& 0.5 & $0.32 \pm 0.01(0.30 \pm 0.02)$ & $1.44 \pm 0.02(0.70 \pm 0.02)$ \\
& 1.0 & $0.22 \pm 0.01(0.31 \pm 0.01)$ & $1.40 \pm 0.01(0.69 \pm 0.01)$ \\
& 2.0 & $0.20 \pm 0.01(0.34 \pm 0.00)$ & $1.39 \pm 0.01(0.66 \pm 0.00)$ \\
\hline Eu@gel & 0.02 & $0.60 \pm 0.09(0.33 \pm 0.07)$ & $1.50 \pm 1.10(0.67 \pm 0.07)$ \\
& 0.05 & $0.37 \pm 0.04(0.28 \pm 0.04)$ & $1.35 \pm 0.04(0.72 \pm 0.04)$ \\
& 0.1 & $0.28 \pm 0.01(0.36 \pm 0.01)$ & $1.30 \pm 0.02(0.64 \pm 0.02)$ \\
& 0.2 & $0.26 \pm 0.01(0.38 \pm 0.01)$ & $1.29 \pm 0.02(0.62 \pm 0.01)$ \\
& 0.5 & $0.18 \pm 0.01(0.38 \pm 0.01)$ & $1.21 \pm 0.01(0.62 \pm 0.01)$ \\
& 1.0 & $0.15 \pm 0.00(0.41 \pm 0.01)$ & $1.13 \pm 0.01(0.59 \pm 0.01)$ \\
& 2.0 & $0.08 \pm 0.00(0.52 \pm 0.00)$ & $0.83 \pm 0.01(0.48 \pm 0.00)$ \\
\hline \hline
\end{tabular}

a) The standard deviation is indicated to the second decimal.

b) Only the component $\tau_{2}$ was observed. 


\section{Figure and chart captions}

Chart 1. (a) Chemical components of the phenol + AOT organogel used in this study. The "strand" structure consists of stacked benzene rings, and the higher-order architectures "fiber" and "fiber bundle" are based on ref [10] for aromatic and alkane solvents. (b) The chemical structure of $\mathrm{Eu}(\mathrm{hfa})_{3}($ phen).

Figure 1. The emission spectra of Eu@liq with $m$-xylene (dashed line) and Eu@gel (solid) detected with $\lambda_{\mathrm{ex}}=355 \mathrm{~nm}$.

Figure 2. The time profiles of the emission intensity $I(t)$ for (a) Eu @liq and (b) Eu@gel, respectively, excited with $\lambda_{\mathrm{ex}}=355 \mathrm{~nm}$. The details of the excitation pulse energy $E_{\mathrm{ex}}$ are shown in the boxes in each panel, where representative values are provided besides the decay profiles for visual aid. The $I(t)$ profiles in panel (a) at $E_{\text {ex }}=0.05-0.2 \mathrm{~mJ}$ mostly overlap. The profile in panel (b) becomes monotonically faster with increasing $E_{\mathrm{ex}}$.

Figure 3. The parameter $J\left(E_{\text {ex }}\right)$ estimated with Eq. (2) as indicative of the photon number emitting from Eu@liq (triangles) and $\mathbf{E u} @ \mathbf{g e l}$ (circles) upon excitation. $J\left(E_{\mathrm{ex}}\right)$ is scaled to $J\left(E_{\mathrm{ex}}=2.0[\mathrm{~mJ}]\right)=$ 1.0 for Eu@gel.

Figure 4. The excitation pulse energy $\left(E_{\mathrm{ex}}\right)$ dependence of the fast component of the pre-exponential factor $\left(A_{1}\right)$ fitted to the emission time profile, $I(t)$, based on Eq. (1). The circles indicate the obtained $A_{1}$ for Eu@ gel, along with the error bars which are from Table 2. The squares stand for $\mathbf{E u} @$ film, the data set of which is taken from ref [11]. 


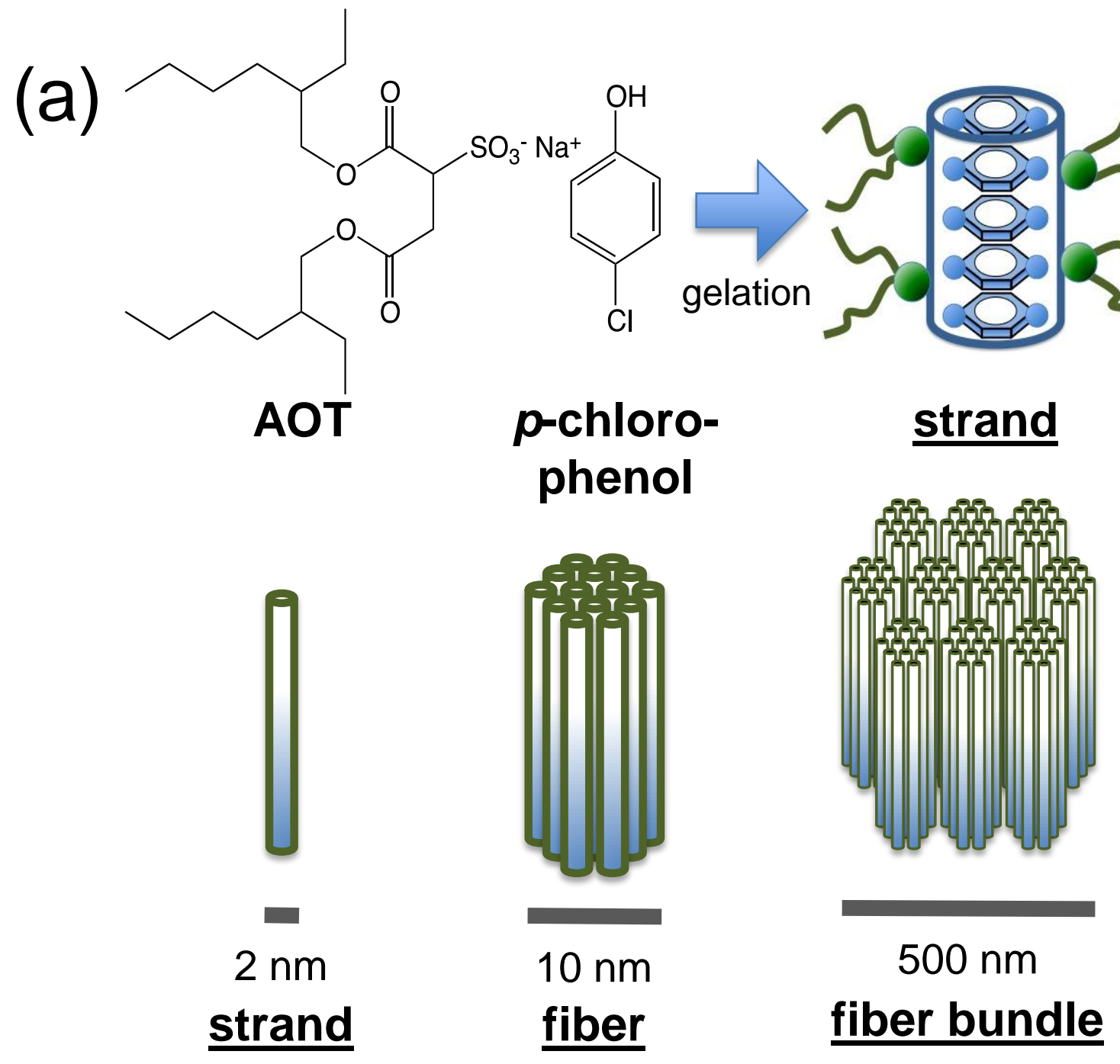

(b)

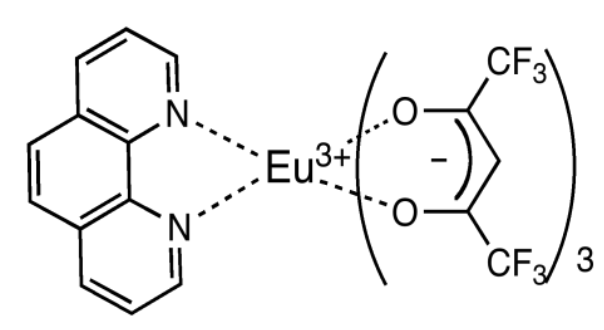

K. Nishiyama and coworkers, Chart. 1 


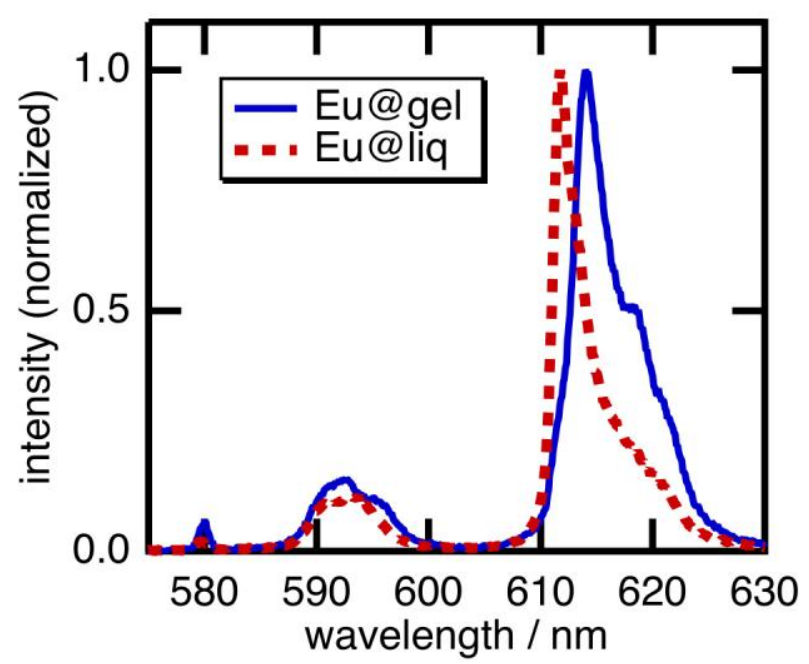

K. Nishiyama and coworkers, Fig. 1.
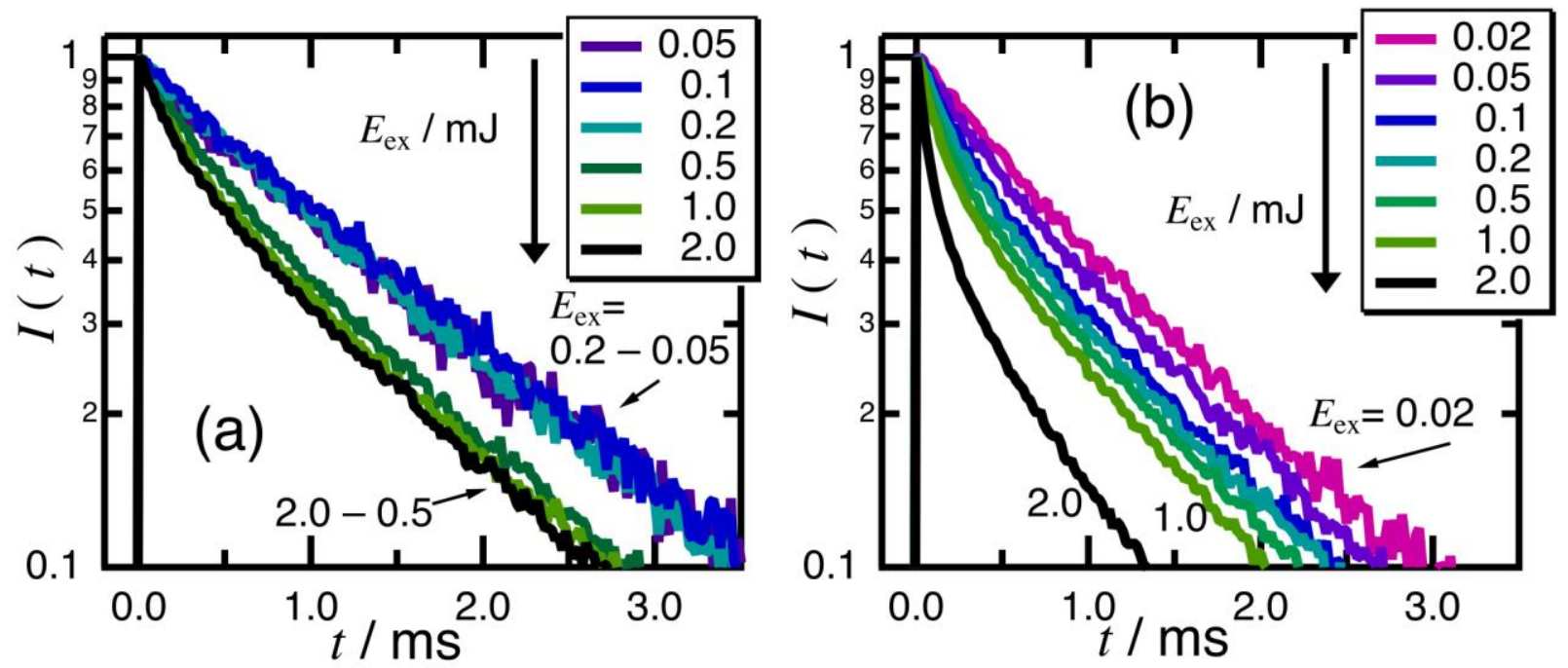

K. Nishiyama and coworkers, Fig. 2. 


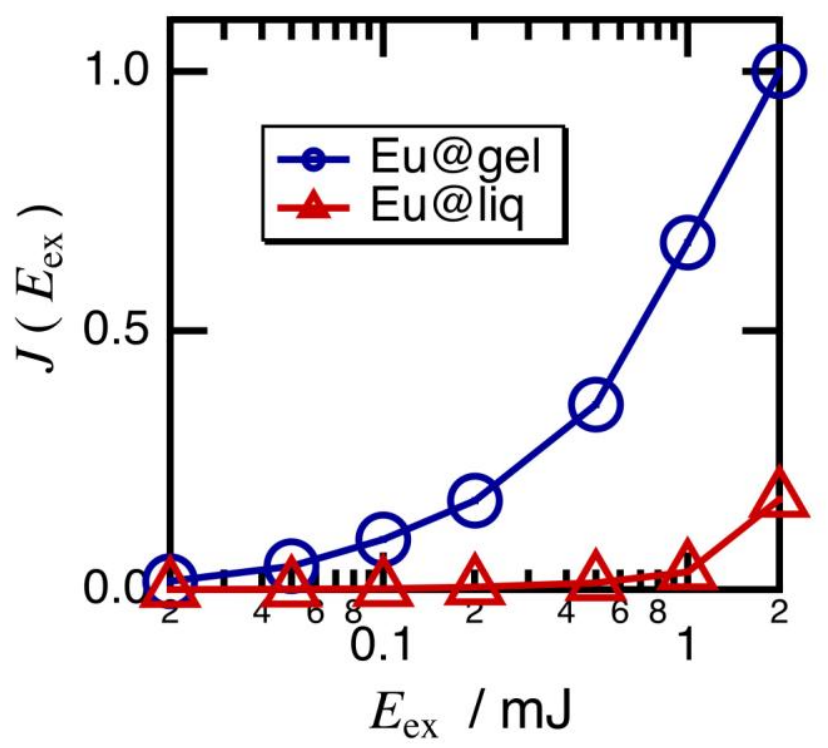

K. Nishiyama and coworkers, Fig. 3.

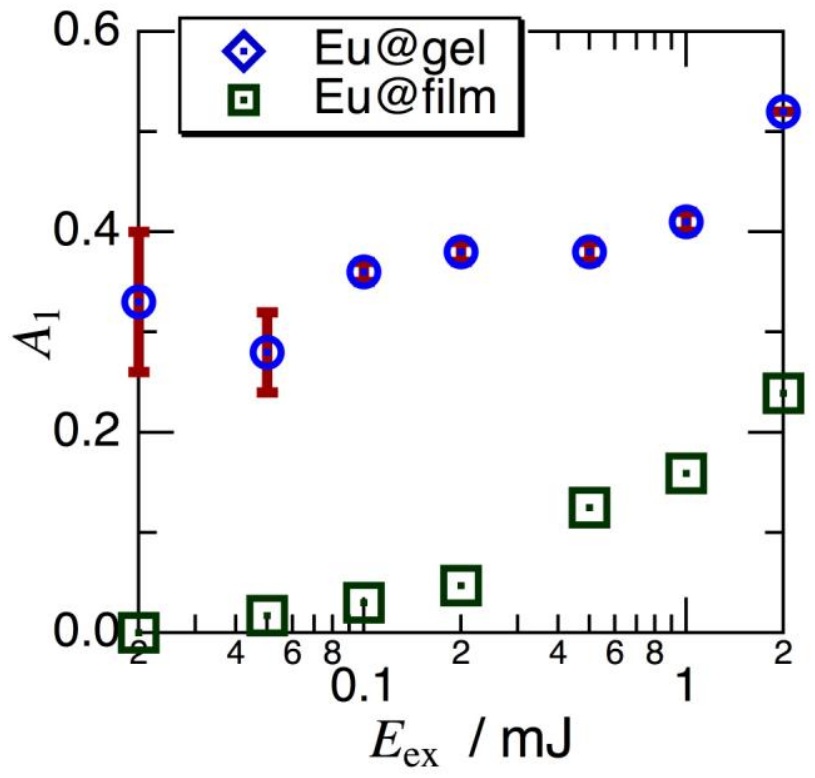

K. Nishiyama and coworkers, Fig. 4. 


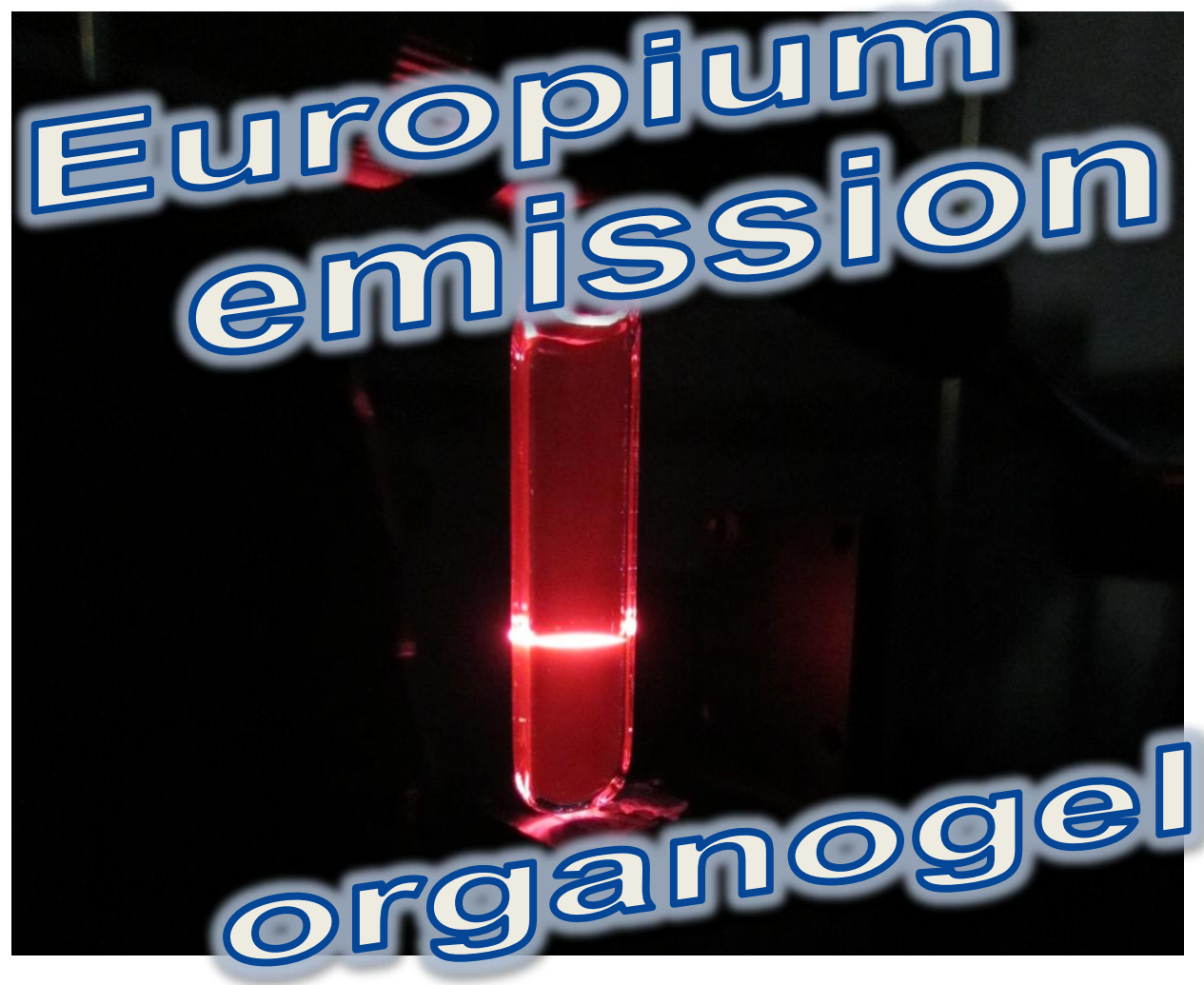

